\title{
MODULE CATEGORIES OF FINITE HOPF ALGEBROIDS, AND SELF-DUALITY
}

\author{
PETER SCHAUENBURG
}

\begin{abstract}
We characterize the module categories of suitably finite Hopf algebroids (more precisely, $\times_{R}$-bialgebras in the sense of Takeuchi [Tak77] that are Hopf and finite in the sense of [Sch00]) as those $k$-linear abelian monoidal categories that are module categories of some algebra, and admit dual objects for "sufficiently many" of their objects.

Then we proceed to show that in many situations the Hopf algebroid can be chosen to be self-dual, in a sense to be made precise. This generalizes a result of Pfeiffer for pivotal fusion categories and the weak Hopf algebras associated to them Pfe09.
\end{abstract}

\section{INTRODUCTION}

The first main result of the present paper is (yet another) variant of the many results that go back at least to the classical Tannaka-Kreinduality. To wit: Which mononidal categories are nice enough to admit the reconstruction of a Hopf algebra (or a structure generalizing somewhat the notion of a Hopf algebra), such that the representations (or corepresentations) of that algebra describe well the category? Which monoidal categories are even equivalent to the representation category of the (co)algebra thus reconstructed?

The generalized Hopf algebras that we will be concerned with in the present paper are the $\times$-bialgebras in the sense of Takeuchi [Tak77], with a Hopf structure in the sense of [Sch00]; we will sometimes refer to them as bialgebroids or Hopf algebroids. $\times$-bialgebras can be characterized in terms of monoidal structures on their bimodule categories [Sch98. To wit, a $\times_{R^{-}}$-bialgebra $H$ is an $R \otimes R^{\text {op }}$-ring such that the category ${ }_{H} \mathfrak{M}$ of left $H$-modules has a monoidal category structure for which the underlying functor to the category of $R$-bimodules (with the tensor product over $R$ ) is a monoidal functor.

1991 Mathematics Subject Classification. 16Txx,18D10.

Key words and phrases. Finite tensor category, fusion category, Hopf algebroid, weak Hopf algebra, self-duality.

Research partially supported through a FABER Grant by the Conseil régional de Bourgogne. 
When is a $\times_{R^{-}}$-bialgebra $H$ Hopf? Assume that $R=k$ is a field, so $H$ is an ordinary bialgebra, and assume that $H$ is finite dimensional; then $H$ is Hopf if and only if the category ${ }_{H} \mathfrak{m}$ of finitely generated $H$ modules is rigid, that is, finitely generated modules admit dual objects. In the general case the follwing answer is given in |Sch00|: $H$ is Hopf if and only if the underlying monoidal functor from $H$ to the monoidal category of $R$-bimodules preserves inner hom-functors. While we hope that this is a satisfactory answer for some purposes, there is a clear disadvantage: The criterion is not intrinsic to the monoidal category, but rather involves the underlying functor, while the condition for a finite dimensional bialgebra over a field only involves the category itself. However, for the general case the requirement that the category $\mathcal{C}$ be rigid is certainly too strong: Not even the category of finitely generated modules over a finitely generated projective $k$-Hopf algebra $H$ over a commutative ring $k$ is rigid, simply because not every finitely generated $k$-module is projective. On the other hand, the category of those $H$ modules that are finitely generated projective $k$-modules is not abelian (See nonetheless [Hái08] for situations where abelian categories of rigid comodules of $\times$-Hopf algebras are treated).

We propose to look instead at categories of modules that are monoidal, and "sufficiently" rigid; this means one of the following equivalent conditions: Every object is the union of subobjects that are epimorphic images of rigid objects; Every object is the colimit of rigid objects; Every finitely generated projective object is rigid; There is a rigid progenerator.

With this definition we can easily show that a $\times_{R}$-bialgebra is Hopf and finite (in the sense of [Sch00], that is, finitely generated projective with respect to a certain one of its four $R$-module structures) if and only if its module category is sufficiently rigid (proposition 4.1).

Now let $\mathcal{C}$ be an abelian monoidal category. When is it the category of modules of a $\times_{R}$-bialgebra or $\times_{R}$-Hopf algebra $H$ ? Clearly tensor products in $\mathcal{C}$ need to be right exact. Moreover, it is necessary to find a monoidal functor to a category of bimodules over an algebra $R$, which will correspond to the underlying functor ${ }_{H} \mathfrak{M} \rightarrow{ }_{R} \mathfrak{M}_{R}$ whose existence characterizes $\times_{R}$-bialgebras. The functor we use in the present paper appears already in the works of Szlachányi [Szl05], Hai Phung [Hái02], and in a special case Hayashi [Hay99]: Assuming that $\mathcal{C}$ is a category of modules, say over $R$, a monoidal functor to $R$-bimodules can be obtained since tensoring with a fixed object gives rise to a right exact endofunctor of the category of $R$-modules, and thus to an $R$-bimodule. We show in theorem 4.2 that via this functor every sufficiently rigid 
monoidal category of modules is monoidally equivalent to the category of modules over a $\times$-Hopf algebra.

We should haste to admit that we have not checked whether theorem 4.2 can be deduced from the very general techniques in BLV11. But we would like to stress that our proof is in fact rather elementary (assuming some familiarity with Morita theory and the basic characterization of $\times$-Hopf algebras). The reason is that we bypass entirely the more general questions as to how to reconstruct a $\times_{R}$-bialgebra from a monoidal functor to $R$-bimodules, or when the original category is equivalent to that of (co-)modules over the reconstructed object. Instead, we make use of the special features of the particular functor under consideration, which allows us to resort directly to Morita theory. We note that in combination with the classical results characterizing module categories among abelian categories, we arrive at an entirely intrinsic characterization of the module categories of finite $\times$-Hopf algebras.

The second main result of the paper generalizes Pfeiffer's theorem Pfe09 on self-duality of pivotal fusion categories. As a special case of theorem 6.5, any fusion category is the representation category of a weak Hopf algebra which is self-dual. More generally every finite tensor category is the representation category of a $\times$-Hopf algebra which is self-dual, and we will discuss some other situations where we know that a self-dual $\times$-Hopf algebra can be chosen for other tensor categories. In fact the main difficulty of the proof lies in clarifying what it should mean for a $\times$-Hopf algebra to be self-dual. The general theory of duality for (suitably finite) $\times$-bialgebras developed in [Sch00] does not (and cannot) provide a dual $\times_{R}$-bialgebra for a $\times_{R^{-}}$bialgebra $H$, but rather a skew dual $H^{\vee}$ which is an analog of the dual of the coopposite of $H$ in the case of ordinary bialgebras. However, for $\times$-Hopf algebras, passing to opposites or coopposites is not such an easy matter: The

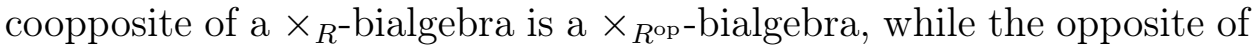
a $\times$-bialgebra may not be a $\times$-bialgebra at all. Thus, to make sense of self-duality, some manipulations with the base ring $R$ of a $\times{ }_{R}$-bialgebra are necessary, and theorem 6.5 gives conditions when a category $\mathcal{C}$ is the module category of a self-dual $\times$-Hopf algebra.

\section{Definitions And Preliminaries}

Throughout the paper we will quite freely use the language of monoidal categories, suppressing the associativity constraints in view of the wellknown coherence results. A general reference is [Kas95]. 
If $\mathcal{C}$ is a monoidal category, we denote by $\mathcal{C}^{\text {rev }}$ the category with reversed order in tensor products (while $\mathcal{C}^{\text {op }}$ is the dual category in which the direction of morphisms is reversed).

We denote the neutral object of a monoidal category $\mathcal{C}$ by $I$. The left dual of an object $X \in \mathcal{C}$, if it exists, is denoted by $X^{*}$, it is equipped by definition with evaluation and coevaluation morphisms

$$
\text { ev }: X^{*} \otimes X \rightarrow I \quad \text { and } \quad \mathrm{db}: I \rightarrow X \otimes X^{*}
$$

An object admitting a left dual will be called left rigid, a category in which every object admits a left dual is also called left rigid.

A right dual of $X$ (which is the same as a left dual in $\mathcal{C}^{\text {rev }}$ ) will be denoted ${ }^{*} X$. A right rigid category has every object right rigid, i. e. admitting a right dual.

We note that in the monoidal category of bimodules over a ring $R$ (with the tensor product over $R$ ) a bimodule is left rigid if and only if it is finitely generated projective as right $R$-module.

An object (a category) will be called rigid if it is left and right rigid.

A right inner hom-functor $\underline{\operatorname{hom}}(X,-)$ in $\mathcal{C}$ is a right adjoint to the endofunctor $-\otimes X$, a left inner hom-functor is a right adjoint to $X \otimes-$. If $X$ is left rigid, then $-\otimes X^{*}$ is a right inner hom-functor. If a right inner hom-functor hom $(X,-)$ exists, and the canonical morphism $Y \otimes$ $\underline{\operatorname{hom}}(X,-) \rightarrow \underline{\operatorname{hom}}(X, Y)$ defined by the adjunction is an isomorphism for all $Y \in \mathcal{C}$, then $X$ is left rigid.

A monoidal functor $(F, \xi): \mathcal{C} \rightarrow \mathcal{D}$ between monoidal categories $\mathcal{C}$ and $\mathcal{D}$ consists of a functor, a natural isomorphism $\xi: F(X \otimes Y) \rightarrow$ $F(X) \otimes F(Y)$ (often suppressed) and an isomorphism $\xi_{0}: F(I) \rightarrow I$ (very often suppressed) that satisfy coherence conditions with the associativity constraints of $\mathcal{C}$ and $\mathcal{D}$.

It is well-known that monoidal functors preserve dual objects.

A monoidal category equivalence is a monoidal functor that is a category equivalence. We will call a monoidal functor $\mathcal{C} \rightarrow \mathcal{D}^{\text {rev }}$ (or equivalently $\mathcal{C}^{\text {rev }} \rightarrow \mathcal{D}$ ) a rev-monoidal functor. If $\mathcal{C}$ and $\mathcal{D}$ are revmonoidally equivalent, we will write $\mathcal{C} \stackrel{\text { rev }}{\cong} \mathcal{D}$.

Let $(F, \xi): \mathcal{C} \rightarrow \mathcal{D}$ be a monoidal functor. The left weak centralizer $\mathcal{W}_{\ell}(F)$ of $F$ is the category whose objects are pairs $(X, \tilde{\tau})$ in which $X$ is an object of $\mathcal{D}$, and $\tilde{\tau}=\tilde{\tau}_{X, V}: X \otimes F(V) \rightarrow F(V) \otimes X$ is a natural 
transformation making

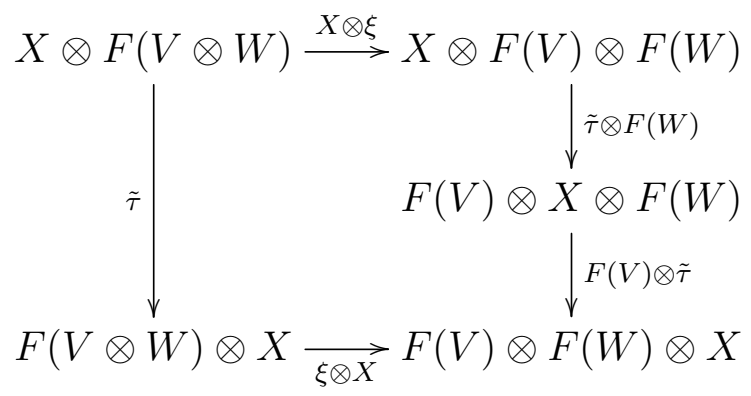

commute and satisfying $\tilde{\tau}=\mathrm{id}: X \otimes F(I) \rightarrow F(I) \otimes X$.

The left weak centralizer is a monoidal category, the tensor product of $X$ and $Y$ being the tensor product $X \otimes Y$ in $\mathcal{D}$ endowed with

$$
\tilde{\tau}=(X \otimes Y \otimes \mathcal{F}(V) \stackrel{X \otimes \tilde{\tau}}{\longrightarrow} X \otimes \mathcal{F}(V) \otimes Y \stackrel{\tilde{\tau} \otimes Y}{\longrightarrow} \mathcal{F}(V) \otimes X \otimes Y)
$$

The right weak centralizer $\mathcal{W}_{r}(F)$ is defined similarly. The (left) centralizer $\mathcal{Z}(F)=\mathcal{Z}_{\ell}(F)$ consists of those objects $(X, \tilde{\tau})$ in the left weak centralizer where $\tilde{\tau}$ is an isomorphism. It is naturally equivalent to the right centralizer. Centralizers and weak centralizers were introduced by Majid Maj91, who calls the right weak centralizer of $F$ the dual of the functored category $(\mathcal{C}, F)$.

A left $\mathcal{C}$-category $\mid$ Par77 $]$, or module category over $\mathcal{C}$ is a category $\mathcal{D}$ with an action of $\mathcal{C}$, that is, a functor $\mathcal{C} \times \mathcal{D} \rightarrow \mathcal{D}$ with an associativity constraint coherent with the associativity constraint of $\mathcal{C}$. Similarly one defines right $\mathcal{C}$-categories and $\mathcal{C}$ - $\mathcal{C}$-bicategories. We note that a tensor functor $\mathcal{F}: \mathcal{C} \rightarrow \mathcal{D}$ endows $\mathcal{D}$ with a natural structure of right $\mathcal{C}$-category, and that the left centralizer of $\mathcal{F}$ is equivalent to the category of right $\mathcal{C}$-endofunctors of $\mathcal{C}$. In this way Majid's notion of duals of functored categories is intimately related to the theory of duals of fusion categories with respect to module categories, or weak Morita equivalence [Müg03b; Müg03a; Müg10; ENO05; Ost03]

We fix throughout a commutative base ring $k$. Algebras, tensor products, etc. are over $k$. We will take the liberty to write $v \otimes w \in V \otimes W$ for a general element of the tensor product of two $k$-modules (or a more general tensor product) even when we do not expect the tensor in question to be simple. For $k$-algebras $A, B$ we write $A \mathfrak{M}, \mathfrak{M}_{B}$, $\mathfrak{M}_{B}$ for the categories of left $A$-modules, right $B$-modules, and $A$ - $B$ bimodules. We use ${ }_{A} \mathfrak{m}$ etc. for the subcategories of finitely generated modules.

We refer to [Tak77; Böh09; Sch98; Sch00] for the axiomatics of $\times_{R^{-}}$ bialgebras and $\times_{R}$-Hopf algebras; we will sometimes also refer to them 
as bialgebroids and Hopf algebroids, in spite of the fact that variants of the definition have been circulating under these names. The most awkward feature of the term $\times_{R}$-bialgebra being that it already contains a choice of "base algebra" $R$, we will sometimes (as already in the introduction) use the term $\times$-bialgebra.

Let $R$ be a $k$-algebra. Conceptually, a $\times_{R^{-}}$-bialgebra is a $k$-algebra $H$ with a $k$-linear monoidal category structure on the module category $H \mathfrak{M}$, given in such a way that an underlying functor to the category of $R$-bimodules (with tensor product over $R$ ) is monoidal. More precisely write $\bar{R}=R^{\text {op }}$ for the opposite $k$-algebra, and $R^{e}=R \otimes \bar{R}$ for the enveloping algebra. We can identify $R_{\mathfrak{M}_{R}}=R_{e} \mathfrak{M}$. An equivalent characterization of the notion of $\times_{R}$-bialgebra introduced by Takeuchi

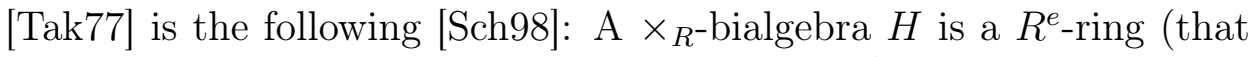
is, an algebra with an algebra map $\left.\iota: R^{e} \rightarrow H\right)$, and endowed with a monoidal category structure on the module category ${ }_{H} \mathfrak{M}$ such that the functor $H_{H} \mathfrak{M} \rightarrow{ }_{R}^{e} \mathfrak{M}$ induced by $\iota$ is monoidal. The axiomatics of

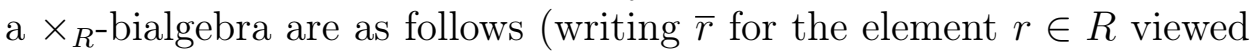
in the opposite algebra $\bar{R}$, and suppressing $\iota): H$ is equipped with a comultiplication, a $R^{e}$-ring homomorphism $\Delta: H \rightarrow H \times_{R} H$, where

$$
\begin{aligned}
H \diamond H & :=H \otimes H / k-\operatorname{span}\{\bar{r} g \otimes h-g \otimes r h \mid r \in R, g, h \in H\} \\
H \times_{R} H & :=\{g \otimes h \in H \diamond H \mid \forall r \in R: g \bar{r} \otimes h=g \otimes h r\},
\end{aligned}
$$

and the latter is a $R^{e}$-ring with $\iota(r \otimes \bar{s})=r \otimes \bar{s}$ We write $\Delta(h)=$ $h_{(1)} \otimes h_{(2)}$. Comultiplication is coassociative in the sense that $\Delta\left(h_{(1)}\right) \otimes$ $h_{(2)}=h_{(1)} \otimes \Delta\left(h_{(2)}\right)$, where we deliberately omitted discussing where this equality takes place. The counit is a certain map of $R^{e}$-rings $\epsilon: H \rightarrow \operatorname{End}(R)$.

If $M, N \in{ }_{H} \mathfrak{M}$, then their tensor product $M \diamond N$ in ${ }_{R^{e}} \mathfrak{M}$ is an $H$ module by $h(m \otimes n)=h_{(1)} m \otimes h_{(2)} n$, and $R \in R^{e} \mathfrak{M}$ is an $H$-module by $h \cdot r=\epsilon(h)(r)$.

A left $H$-comodule is a $R$ - $R$-bimodule $M$ equipped with a structure map $\lambda: M \rightarrow H \times_{R} M$, written $\lambda(m)=m_{(-1)} \otimes m_{(0)}$, such that $\Delta\left(m_{(-1)}\right) \otimes m_{(0)}=m_{(-1)} \otimes \lambda(m)$, and $\epsilon\left(m_{(-1)}\right)(1) m_{(0)}=m$. We write ${ }^{H} \mathfrak{M}$ for the category of left $H$-comodules. The tensor product $M \otimes \otimes_{R} N$ of $M, N \in{ }^{H} \mathfrak{M}$ is again an $H$-comodule by $\lambda(m \otimes n)=$ $m_{(-1)} n_{(-1)} \otimes m_{(0)} \otimes n_{(0)}$.

A $\times_{R^{-}}$-bialgebra $H$ is said to be a $\times_{R^{-H}}$ Hopf algebra if the map

$$
H \underset{\bar{R}}{\otimes} H \ni g \otimes h \mapsto g_{(1)} \otimes g_{(2)} h \in H \diamond H
$$


is an isomorphism. As shown in [Sch00, Thm. 3.5] this is equivalent to the condition that the underlying functor ${ }_{H} \mathfrak{M} \rightarrow{ }_{R} \mathfrak{M} \mathfrak{M}$ preserves inner hom-functors.

A $\times_{R^{-}}$bialgebra $H$ is said to be left finite if it is finitely generated projective as left $\bar{R}$-module, and right finite if it is finitely generated projective as left $R$-module. If $H$ is left finite, then $H^{\vee}:=\operatorname{Hom}_{\bar{R}-}(H, R)$ is a $\times_{R}$-bialgebra such that

$$
\begin{aligned}
& \langle\xi \mid g h\rangle=\left\langle\xi_{(1)} \mid g \overline{\left\langle\xi_{(2)} \mid h\right\rangle}\right\rangle=\left\langle\overline{\left\langle\xi_{(2)} \mid h\right\rangle} \xi_{(1)} \mid g\right\rangle \\
& \langle\xi \zeta \mid h\rangle=\left\langle\xi \mid\left\langle\zeta \mid h_{(1)}\right\rangle h_{(2)}\right\rangle=\left\langle\xi\left\langle\zeta \mid h_{(1)}\right\rangle \mid h_{(2)}\right\rangle,
\end{aligned}
$$

where $\langle\xi \mid h\rangle=\xi(h)$ for $\xi \in H^{\vee}$ and $h \in H$. Thus, if $R=k, H^{\vee}$ is the dual $k$-bialgebra of the coopposite of $H$. The skew dual $H^{\vee}$ can be characterized through a certain equivalence of monoidal categories ${ }^{H} \mathfrak{M} \cong{ }_{H} \vee \mathfrak{M}$.

Finally, note that the opposite of a $\times_{R}$-bialgebra is generally not a $\times$ bialgebra (but see [Böh09] for alternative notions of bialgebroids stable under taking opposites), while the coopposite $H^{\text {cop }}$ of a $\times_{R^{-b i a l g e b r a ~}}$ is an $R^{\text {op}}$-bialgebra; the category ${ }_{H}{ }^{\text {cop }} \mathfrak{M}$ is rev-equivalent to ${ }_{H} \mathfrak{M}$.

\section{Sufficiently Rigid CATEGORIES}

We propose to study a class of abelian monoidal categories which are not rigid, but have "enough" dualizable objects to correspond well to (generalized) Hopf algebras among (generalized) bialgebras.

Definition 3.1. Let $\mathcal{C}$ be an abelian monoidal category. An object of $\mathcal{C}$ is left rigidly generated if it is the epimorphic image of a left rigid object of $\mathcal{C}$. An object is left ind-rigid if it is the directed limit of rigid subobjects. An object is left ind-rigidly generated if it is the directed limit of rigidly generated subobjects. The category $\mathcal{C}$ is left rigidly generated (resp. ind-rigid, resp. ind-rigidly generated) if every object of $\mathcal{C}$ is.

We note that a left ind-rigidly generated finitely generated object of an abelian monoidal category is left rigidly generated.

Examples 3.2. Let $k$ be a commutative ring.

(1) The category of $k$-modules is ind-rigidly generated.

(2) Let $R$ be a $k$-algebra finitely generated projective as $k$-module. Then the category of $R$-bimodules is ind-rigidly generated.

(3) Assume $k$ is a field and $R$ is a $k$-algebra. Then the subcategory of ${ }_{R} \mathfrak{M}_{R}$ of those bimodules that are finite dimensional over $k$ is rigidly generated. 
(4) Let $H$ be a $k$-quasi-Hopf algebra finitely generated projective as $k$-module. Then ${ }_{H} \mathfrak{M}$ is a left ind-rigidly generated monoidal category (which is even easier to see after lemma 3.5.)

(5) Let $k$ be a field, and $H$ a coquasi-Hopf algebra. Then the category $\mathfrak{M}^{H}$ of $H$-comodules is left ind-rigid.

(6) Let $k$ be a field, $H$ a coquasi-Hopf algebra, and $A$ a finitedimensional algebra in the category $\mathfrak{M}^{H}$. Then the category $A_{A} \mathfrak{M}_{A}^{H}$ of $A$-bimodules in $\mathfrak{M}^{H}$ is ind-rigidly generated.

(7) The category of $k$-modules graded by a group $G$ is ind-rigidly generated; similarly every ind-rigidly generated category gives rise to a category of $G$-graded objects in it, which is also indrigidly generated.

Lemma 3.3. Let $\mathcal{C}$ be an abelian monoidal category. If $M \in \mathcal{C}$ is the colimit of a diagram of left rigid objects, then $M$ is left rigidly generated.

Proof. Neglecting the arrows of the diagram, $M$ is the image of the direct sum of its objects, and thus the directed limit of the images of direct sums of finite collections of those objects; these finite sums are left rigid.

Lemma 3.4. Let $\mathcal{C}$ be an abelian monoidal category, $X$ a left rigid object of $\mathcal{C}$, and $Y$ a direct summand of $X$. Then $Y$ is left rigid.

In particular any projective and left rigidly generated object, as well as any finitely generated projective left ind-rigidly generated object is left rigid.

Proof. $Y$ is the image of some projector $p \in \operatorname{End}_{\mathcal{C}}(X)$. Take $Z$ to be the image of $p^{*} \in \operatorname{End}_{\mathcal{C}}\left(X^{*}\right)$. Then $Z$ is a left dual for $Y$ with the evaluation and coevaluation morphisms defined by

$$
\begin{aligned}
& \mathrm{ev}: Z \otimes Y \stackrel{\iota \otimes \iota}{\longrightarrow} X^{*} \otimes X \stackrel{\mathrm{ev}}{\longrightarrow} I \\
& \mathrm{db}: I \stackrel{\mathrm{db}}{\longrightarrow} X \otimes X^{*} \stackrel{\pi \otimes \pi}{\longrightarrow} Y \otimes Z
\end{aligned}
$$

Lemma 3.5. Let $\mathcal{C}$ be an abelian monoidal category. Assume that every object of $\mathcal{C}$ is the direct limit of its finitely generated subobjects, and that every finitely generated object is the epimorphic image of a finitely generated projective object. Then the following are equivalent:

(1) $\mathcal{C}$ is left ind-rigidly generated.

(2) Every object of $\mathcal{C}$ is the colimit of a diagram of left rigid objects.

(3) Every finitely generated projective object of $\mathcal{C}$ is left rigid. 
Proof. $(1) \Rightarrow(3)$ If $M$ is finitely generated, and left ind-rigidly generated, then $M$ is left rigidly generated, so there is an epimorphism $V \rightarrow M$ from a left rigid object $V$. If $M$ is moreover projective, then $M$ is a direct summand of $V$ and thus left rigid.

$(3) \Rightarrow 2$ since our assumptions on $\mathcal{C}$ imply that every object of $\mathcal{C}$ is the colimit of a diagram of finitely generated projective objects.

$(2) \Rightarrow(1)$ was proved in lemma 3.3 .

Note that our assumptions on $\mathcal{C}$ are clearly satisfied if $\mathcal{C}$ happens to be a module category. If $\mathcal{C}$ is noetherian, the condition that every finitely generated object is the image of a finitely generated projective can be rephrased as saying that the (abelian) subcategory of finitely generated objects has enough projectives.

Definition 3.6. We will say that $\mathcal{C}$ is sufficiently left rigid if the assumptions and the equivalent conditions in lemma 3.5 hold.

If the assumptions of lemma 3.5 are not met, then we hesitate to decide whether "left ind-rigidly generated" or "every object is the colimit of left rigid ones" makes the better definition of "sufficiently rigid". The former is a step-by-step weakening of the condition of rigidity, the latter seems more categorically natural.

Let $\mathcal{C}$ be an abelian monoidal category that contains a left rigid generator $G$. Then every finitely generated projective object of $G$ is the epimorphic image and hence a direct summand of a finite sum of copies of $G$, and thus left rigid.

If $\mathcal{C}$ has arbitrary colimits, and contains a progenerator (that is, a small projective generator), then $\mathcal{C}$ is equivalent to a category of modules by a theorem of Gabriel and Mitchell; see [Mit65]. Thus we obtain the following characterization:

Lemma 3.7. Let $\mathcal{C}$ be an abelian monoidal category. The following are equivalent:

(1) $\mathcal{C}$ is cocomplete and has a left rigid progenerator.

$(2) \mathcal{C}$ is a sufficiently left rigid category of modules.

Finally, we note how the presence of (sufficiently many) dual objects affects the (weak) centralizers of a monoidal functor $\mathcal{F}: \mathcal{C} \rightarrow \mathcal{D}$ : It is well-known that if $V \in \mathcal{C}$ is right rigid, and $\left(X, \tilde{\tau}_{X,-}\right) \in \mathcal{W}_{\ell}(F)$, then $\tilde{\tau}_{X, V}$ is an isomorphism. In fact the inverse of $\tilde{\tau}_{V, X}$ is the image of $\tilde{\tau}_{* V, X}$ under the canonical isomorphism

$$
\mathcal{D}\left(X \otimes \mathcal{F}\left({ }^{*} V\right), \mathcal{F}\left({ }^{*} V\right) \otimes X\right) \cong \mathcal{D}(\mathcal{F}(V) \otimes X, X \otimes \mathcal{F}(V)) .
$$

In particular, if $\mathcal{C}$ is right rigid then $\mathcal{W}_{\ell}(F)=\mathcal{Z}(F)$ 
If $\mathcal{F}$ is a right exact functor between abelian categories, then one can conclude that $\tilde{\tau}_{X, V}$ is an isomorphism for all right ind-rigidly generated objects. Thus

Lemma 3.8. Let $F: \mathcal{C} \rightarrow \mathcal{D}$ be a right exact monoidal functor between abelian monoidal categories. If $\mathcal{C}$ is right ind-rigidly generated, then the inclusion functor $\mathcal{Z}(F) \rightarrow \mathcal{W}_{\ell}(F)$ is the identity.

\section{Module CAtegories of Finite Hopf Algebroids}

In this section we will give an intrinsic characterization of the module categories of finite $\times$-Hopf algebras. We start by characterizing the finite $\times$-Hopf algebras among all $\times$-bialgebras by intrinsic properties of their module categories.

Proposition 4.1. Let $B$ be a k-algebra, and $H a \times_{B}$-bialgebra. The following are equivalent:

(1) $H$ is a $\times_{B}$-Hopf algebra and finitely generated projective as a left $\bar{B}$-module.

(2) $H$ is a left rigid object in $H \mathfrak{M}$.

(3) The category ${ }_{H} \mathfrak{M}$ is sufficiently left rigid.

If $B$ is noetherian, these are further equivalent to ${ }_{H} \mathfrak{m}$ being rigidly generated.

Proof. It was shown in Sch00 that ${ }_{H} \mathfrak{M}$ admits right inner hom-functors $\underline{\operatorname{hom}}(M, N)$, and that $H$ is $\times_{B}$-Hopf if and only if the underlying functor ${ }_{H} \mathfrak{M} \rightarrow{ }_{B}{ }^{e} \mathfrak{M}$ preserves these. Inspecting the proof of [Sch00, Thm. 3.5] one sees that it suffices already that the underlying functor preserves the hom-functors hom $(H, P)$ for $P \in{ }_{H} \mathfrak{M}$. On the other hand an object $X$ in a monoidal category with neutral object $B$ admitting right inner hom-functors is left rigid if and only if all the canonical morphisms

$$
C: \underline{\operatorname{hom}}(X, P) \rightarrow P \diamond \underline{\operatorname{hom}}(X, I)
$$

are isomorphisms. Specialize $X=H \in{ }_{H} \mathfrak{M}$. If the underlying functor preserves the inner hom-functors $\underline{\operatorname{hom}}(H, P)$ and $H$ is finitely generated projective as a left $\bar{B}$-module, then $C$ is an isomorphism because this is the case in ${ }_{B} \mathfrak{M}^{\mathrm{M}}$. On the other hand, if $H$ is left rigid in ${ }_{H} \mathfrak{M}$ then $C$ is an isomorphism, and $\underline{\operatorname{hom}}(H, I)$ is the left dual of $H$, hence preserved by the underlying functor; thus, the instance hom $(H, P)$ of the homfunctor is also preserved. This shows the equivalence of (1) and (2).

If $H \mathfrak{M}$ is ind-rigidly generated, then $H$ is a left rigid object by Lemma 3.4.

The equivalence of (2) and (3) is already covered in lemma 3.7. 
As an obvious special case we note that the category ${ }_{H} \mathfrak{M}$ of modules over an ordinary $k$-bialgebra is therefore sufficiently rigid if and only if $H$ is a Hopf algebra, and finitely generated projective as $k$-module.

Now we will proceed to show that any sufficiently rigid $k$-linear monoidal category of modules is the category of modules over a Hopf algebroid, while proposition 4.1 assumed that the category is already given as the module category of a bialgebroid.

Let $A$ be a $k$-algebra, and denote by $\mathcal{C}={ }_{A} \mathfrak{M}$ the abelian category of left $A$-modules. Suppose that $\mathcal{C}$ is a $k$-linear abelian monoidal category with respect to some tensor product denoted $\diamond$ which we can safely assume to be strict. We assume further that $\diamond$ preserves colimits in each argument, and that $\mathcal{C}$ is left ind-rigidly generated.

Note that the regular $A$-module $A \in \mathcal{C}$ is a finitely generated projective object, and thus left rigid by Lemma 3.4 .

By Watts' theorem, for every $X \in \mathcal{C}$ there are $A$-bimodules $L(X), R(X) \in$ $A^{\mathfrak{M}_{A}}$ such that $X \diamond Y=L(X) \otimes Y$ and $Y \diamond X=R(X) \otimes Y$ for every $Y \in A \mathfrak{M}$, namely $L(X)=X \diamond A$ and $R(X)=A \diamond X$. Clearly $L$ and $R$ extend to additive functors $L, R: \mathcal{C} \rightarrow{ }_{A} \mathfrak{M}_{A}$. Moreover, $L$ is a monoidal functor, and $R: \mathcal{C}^{\text {rev }} \rightarrow{ }_{A} \mathfrak{M}_{A}$ is a monoidal functor. Also, $L$ (and similarly $R$ ) is faithful since $L(X) \otimes I=X \diamond I=X$.

Further we can compute

$$
L(X)=L(X) \otimes_{A} A=X \diamond A=R(A) \otimes_{A} X
$$

and similarly $R(X)=L(A) \otimes_{A} X$, from which we can conclude that both $R$ and $L$ are right exact functors. Moreover, $L(A)$, as the image of a left rigid object under a monoidal functor, is left rigid and thus finitely generated projective as a right $A$-module.

Let $\Lambda$ be some index set and $A^{(\Lambda)} \stackrel{\nu}{\rightarrow} I$ an epimorphism from a possibly infinite direct sum of copies of $A$ onto $I$. Applying the right exact functor $L$ gives an epimorphsim $R(\nu): L(A)^{(\Lambda)} \rightarrow A$, and since $A$ is finitely generated, a finite subset of $n$ summands suffices to obtain an epimorphism $\nu^{\prime}: L(A)^{n} \rightarrow A$. In particular the right $A$-module $L(A)$ is a progenerator. Let $H=\operatorname{End}_{-A}(L(A))$. Then the Morita theorems imply that

$$
\begin{aligned}
\mathcal{C}={ }_{A} \mathfrak{M} & \longrightarrow{ }_{H} \mathfrak{M} \\
X & \longmapsto L(A){ }_{A} X=R(X)
\end{aligned}
$$

is a category equivalence. Put $B=A^{\text {op }}$. Then we can view $R$ as a monoidal functor $R: \mathcal{C} \rightarrow\left({ }_{A} \mathfrak{M}_{A}\right)^{\text {rev }} \cong{ }_{B} \mathfrak{M}_{B} \cong{ }_{B} \mathfrak{M}$, which factors over a functor ${ }_{H} \mathfrak{M} \rightarrow{ }_{B} \mathfrak{M} \mathfrak{M}$ that commutes with the underlying functors 
to $k$-modules. We are in the situation of [Sch98, Thm. 5.1, Rem. 5.3] and can conclude that $H$ admits a $\times_{B}$-bialgebra structure for which the equivalence $A_{A} \mathfrak{M} \rightarrow{ }_{H} \mathfrak{M}$ is a monoidal equivalence.

Taking into account proposition 4.1 and lemma 3.7, we have shown:

Theorem 4.2. Let $\mathcal{C}$ be a k-linear abelian monoidal category. The following are equivalent:

(1) (a) $\mathcal{C}$ is equivalent, as a $k$-linear category, to the category of modules over a k-algebra.

(b) The tensor product of $\mathcal{C}$ is right exact in each argument.

(c) $\mathcal{C}$ is sufficiently left rigid.

(2) (a) $\mathcal{C}$ is cocomplete.

(b) The tensor product of $\mathcal{C}$ is right exact in each argument.

(c) $\mathcal{C}$ contains a left rigid progenerator.

$(3) \mathcal{C} \cong{ }_{H} \mathfrak{M}$, where $H$ is a left finite $\times$-Hopf algebra.

Remark 4.3. Since the functor $R:{ }_{A} \mathfrak{M} \rightarrow{ }_{A} \mathfrak{M}_{A} \cong{ }_{A \otimes}$ op $\mathfrak{M}$ is given by $R(X)=L(A) \otimes_{A} X$, we know that $L(A)$ is an $A \otimes A^{\text {op }}-A$-bimodule, or an $A-A \otimes A$-bimodule. One right $A$-module structure comes from the fact that $L$ is a functor to the category of bimodules, the other is given by the composition

$$
A^{\mathrm{op}} \cong \operatorname{End}_{A-}(A) \stackrel{L}{\rightarrow} \operatorname{End}_{A-A}(L(A)) .
$$

The same holds for $R(A)$.

Note that moreover $L(A)=R(A) \otimes A=R(A)$ as left $A$-modules. The isomorphism is in fact right $A \otimes A$-linear as well, but exchanges the two types of right $A$-module structures.

\section{WEAK CENTRALIZERS AND DUAL BIALGEBROIDS}

We take a moment to establish the unsurprising fact that the theory of dual categories in the sense of [Maj91] works well with the notion of a dual bialgebroid used in $|\mathrm{Sch} 00|$.

The model case we should keep in mind is the case of a finite dimensional Hopf algebra $H$. The category ${ }_{H} \mathfrak{M}$ has a natural underlying functor to the category of vector spaces. Its left weak centralizer is the category ${ }^{H} \mathfrak{M}$ of $H$-comodules, and inversely the category ${ }_{H} \mathfrak{M}$ is the right weak centralizer of the underlying functor from ${ }^{H} \mathfrak{M}$. We prove the same thing for arbitrary $\times_{R}$-bialgebras and the relevant underlying functors to categories of bimodules.

Only the second part of the following Proposition will be needed in the subsequent sections. Both parts should be considered as supplying an easy standard tool for $\times$-bialgebra theory; the proofs are entirely 
routine, only complicated, as usual for the theory of $\times$-bialgebras, by tedious verifications that all too obvious maps are well defined.

Proposition 5.1. Let $H$ be $a \times_{R}$-bialgebra. For $M \in{ }_{H} \mathfrak{M}$ and $X \in$ ${ }^{H} \mathfrak{M}$ define

$$
\sigma=\sigma_{X N}: X \underset{R}{\otimes} N \ni x \otimes n \mapsto x_{(-1)} n \otimes x_{(0)} \in N \diamond X .
$$

(1) Identifying the underlying $R^{e}$-module of an $H$-module $N$ with the corresponding $R$-bimodule makes $\sigma$ a morphism $X \underset{R}{\otimes} N \rightarrow$ $N \underset{R}{\otimes}$ X. This defines a monoidal category equivalence

$$
{ }_{H} \mathfrak{M} \ni N \mapsto(N, \sigma) \in \mathcal{W}_{r}\left({ }^{H} \mathfrak{M} \rightarrow{ }_{R} \mathfrak{M}_{R}\right)
$$

(2) Identifying the underlying $R$-bimodule of an $H$-comodule $X$ with the corresponding $R^{e}$-module makes $\sigma$ a morphism $X \diamond N \rightarrow$ $N \diamond X$. This defines a monoidal category equivalence

$$
{ }^{H} \mathfrak{M} \ni X \mapsto(X, \sigma) \in \mathcal{W}_{\ell}\left({ }_{H} \mathfrak{M} \rightarrow R^{e} \mathfrak{M}\right)
$$

Proof. We need to check that $\sigma$ is well-defined. Abusing notation, this amounts to the calculation

$$
\begin{aligned}
\sigma(x r \otimes n) & =(x r)_{(-1)} n \otimes(x r)_{(0)} \\
& =x_{(-1)} r n \otimes x_{(0)} \\
& =\sigma(x \otimes r n) .
\end{aligned}
$$

Next, we note that

$$
\begin{aligned}
\sigma(r x \otimes n) & =(r x)_{(-1)} n \otimes(r x)_{(0)} \\
& =r x_{(-1)} n \otimes x_{(0)} \\
& =r \sigma(x \otimes n)
\end{aligned}
$$

and

$$
\begin{aligned}
\sigma(x \otimes \bar{r} n) & =x_{(-1)} \bar{r} n \otimes x_{(0)} \\
& =x_{(-1)} n \otimes x_{(0)} r \\
& =\sigma(x \otimes n) r .
\end{aligned}
$$

Note that the last equation means that for a left $R$-module $T$ we get a map

$$
\sigma \otimes T:(X \underset{R}{\otimes} N) \diamond T \rightarrow(N \diamond X) \underset{R}{\otimes} T=N \diamond(X \underset{R}{\otimes} T) .
$$


The diagram

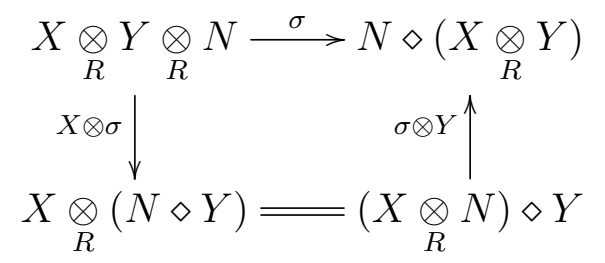

commutes by the calculation

$$
\begin{aligned}
(\sigma \otimes Y)(X \otimes \sigma)(x \otimes y \otimes n) & =(\sigma \otimes Y)\left(x \otimes y_{(-1)} n \otimes y_{(0)}\right) \\
& =x_{(-1)} y_{(-1)} n \otimes x_{(0)} \otimes y_{(0)} \\
& =(x \otimes y)_{(-1)} n \otimes(x \otimes y)_{(0)} \\
& =\sigma(x \otimes y \otimes n)
\end{aligned}
$$

and the diagram

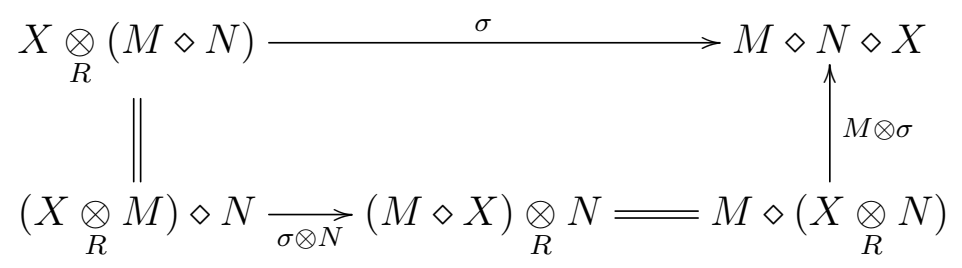

commutes by

$$
\begin{aligned}
(M \otimes \sigma)(\sigma \otimes N)(x \otimes m \otimes n) & =(M \otimes \sigma)\left(x_{(-1)} m \otimes x_{(0)} \otimes n\right) \\
& =x_{(-2)} m \otimes x_{(-1)} n \otimes x_{(0)} \\
& =x_{(-1)}(m \otimes n) \otimes x_{(0)} \\
& =\sigma(x \otimes m \otimes n)
\end{aligned}
$$

These calculations show that the two functors in (1) and (2) are welldefined and monoidal.

Let us show that the functor in (1) is an equivalence. Thus consider $(N, \sigma) \in \mathcal{W}_{r}\left({ }^{H} \mathfrak{M} \rightarrow{ }_{R} \mathfrak{M}_{R}\right)$; consider $N$ as an $R^{e}$-module. This converts $\sigma$ to a natural transformation $\sigma: X \underset{R}{\otimes} N \rightarrow N \diamond X$. Since $H \in{ }^{H} \mathfrak{M}$, we obtain

$$
\mu=\left(H \underset{R}{\otimes} N \stackrel{\sigma}{\rightarrow} N \diamond H \stackrel{N \diamond \epsilon_{0}}{\rightarrow} N\right) .
$$

We write $\mu(\ell \otimes n)=: \ell n$.

Since $\delta_{0}: X \rightarrow H \diamond X$ is left $H$-colinear with

$$
\left(X \stackrel{\delta_{0}}{\rightarrow} H \diamond X \stackrel{\epsilon_{0} \diamond X}{\rightarrow} X\right)=\mathrm{id}_{X}
$$


we have a commutative diagram

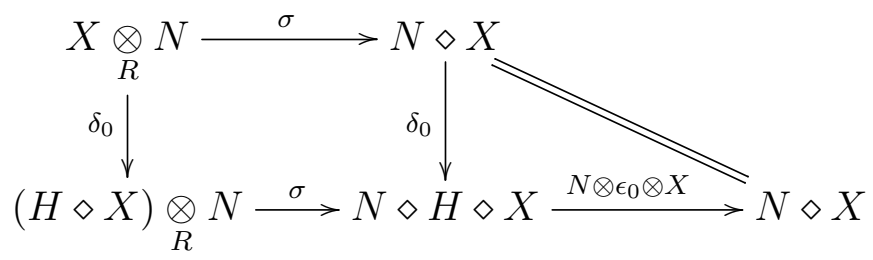

And since $\sigma_{H \diamond X, N}(\ell \otimes x \otimes n)=\sigma_{H N}(\ell \otimes n) \otimes x$ we deduce that

$$
\sigma_{X N}(x \otimes n)=x_{(-1)} n \otimes x_{(0)} .
$$

It follows that

$$
\begin{aligned}
\ell_{(1)}(\bar{r} n) \otimes \ell_{(2)} & =\sigma_{H N}(\ell \otimes \bar{r} n) \\
& =\sigma_{H N}(\ell \otimes n) r \\
& =\ell_{(1)} n \otimes \ell_{(2)} r \\
& =\left(\ell_{(1)} \bar{r}\right) n \otimes \ell_{(2)}
\end{aligned}
$$

and, upon applying $N \diamond \epsilon_{0}$, that $\ell(\bar{r} n)=(\ell \bar{r}) n$. Thus $\mu$ factors over $H \underset{R^{e}}{\otimes} N$. By definition $\mu$ is left $R$-inear, but also

$$
\begin{aligned}
(\bar{r} \ell) n & =\left(N \diamond \epsilon_{0}\right) \sigma_{H N}(\bar{r} \ell \otimes n) \\
& =\left(N \diamond \epsilon_{0}\right)\left((\bar{r} \ell)_{(1)} n \otimes(\bar{r} \ell)_{(2)}\right) \\
& =(N \diamond \epsilon)\left(\ell_{(1)} n \otimes \bar{r} \ell_{(2)}\right) \\
& =\overline{\epsilon_{0}\left(\bar{r} \ell_{(2)}\right)}\left(\ell_{(1)} n\right) \\
& =\bar{r} \overline{\epsilon_{0}\left(\ell_{(2)}\right)}\left(\ell_{(1)} n\right) \\
& =\bar{r}(\ell n)
\end{aligned}
$$

since

$$
\ell n=\left(N \diamond \epsilon_{0}\right)\left(\ell_{(1)} n \otimes \ell_{(2)}\right)=\overline{\epsilon_{0}\left(\ell_{(2)}\right)}\left(\ell_{(1)} n\right) .
$$

Next, consider the left $H$-comodule $H$ and the left $H$-colinear multiplication map $\nabla: H \underset{R}{\otimes} H \rightarrow H$. Naturality of $\sigma$ gives a commutative diagram

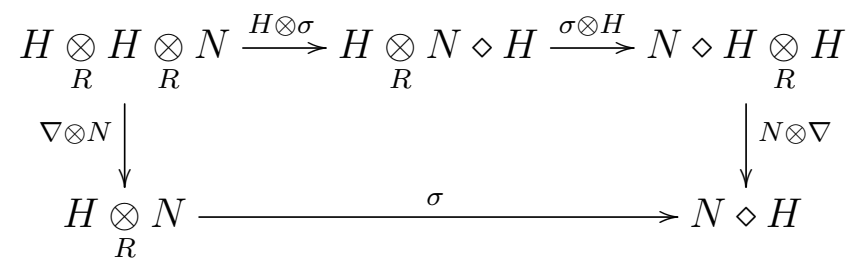


Thus

$$
\begin{aligned}
\left(\ell \ell^{\prime}\right)_{(1)} n \otimes\left(\ell \ell^{\prime}\right)_{(2)} & =\sigma\left(\ell \ell^{\prime} \otimes n\right) \\
& =(N \otimes \nabla)(\sigma \otimes H)(H \otimes \sigma)\left(\ell \otimes \ell^{\prime} \otimes n\right) \\
& =(N \otimes \nabla)(\sigma \otimes H)\left(\ell \otimes \ell^{\prime}{ }_{(1)} n \otimes \ell^{\prime}{ }_{(2)}\right) \\
& =(N \otimes \nabla)\left(\ell_{(1)}\left(\ell_{(1)}^{\prime} n\right) \otimes \ell_{(2)} \otimes \ell^{\prime}{ }_{(2)}\right) \\
& =\ell_{(1)}\left(\ell_{(1)}^{\prime} n\right) \otimes \ell_{(2)} \ell_{(2)}^{\prime} .
\end{aligned}
$$

To this we can apply $N \otimes \epsilon_{0}$ to obtain

$$
\begin{aligned}
\left(\ell \ell^{\prime}\right) n & =\overline{\epsilon_{0}\left(\left(\ell \ell^{\prime}\right)_{(2)}\right)}\left(\ell \ell^{\prime}\right)_{(1)} n \\
& =\overline{\epsilon_{0}\left(\ell_{(2)} \ell_{(2)}\right)} \ell_{(1)}\left(\ell_{(1)}^{\prime} n\right) \\
& =\overline{\epsilon_{0}\left(\ell_{(2)} \epsilon_{0}\left(\ell^{\prime}{ }_{(2)}\right)\right)} \ell_{(1)}\left(\ell^{\prime}{ }_{(1)} n\right) \\
& =\overline{\epsilon_{0}\left(\ell_{(2)}\right)} \ell_{(1)} \overline{\epsilon_{0}\left(\ell^{\prime}{ }_{(2)}\right)}\left(\ell^{\prime}{ }_{(1)} n\right) \\
& =\overline{\ell \epsilon_{0}\left(\ell^{\prime}(2)\right)}\left(\ell_{(1)}^{\prime} n\right) \\
& \left.=\ell \overline{\left(\epsilon_{0}\left(\ell^{\prime}(2)\right)\right.} \ell_{(1)}^{\prime} n\right) \\
& =\ell\left(\ell^{\prime} n\right) .
\end{aligned}
$$

Thus, $N$ is a left $H$-module, and we have found an inverse image of $(N, \sigma)$ under the functor in (1).

To show that the functor in (2) is an equivalence, consider $(X, \sigma) \in$ $\mathcal{W}_{\ell}\left(H_{H} \mathfrak{M} \rightarrow R^{e} \mathfrak{M}\right)$. Identifying $X \in R^{e} \mathfrak{M}$ with the corresponding $R$ bimodule translates $\sigma$ to a map $\sigma: X \underset{R}{\otimes} N \rightarrow N \diamond X$. We define

$$
\delta_{0}=(X \rightarrow X \underset{R}{\otimes} H \stackrel{\sigma}{\rightarrow} H \diamond X)
$$

where the first map sends $x$ to $x \otimes 1$. Write $\delta_{0}(x)=x_{(-1)} \otimes x_{(0)}$. For $n \in N \in{ }_{H} \mathfrak{M}$, we use naturality of $\sigma$ with respect to the $H$-module map $\hat{n}: H \rightarrow N$ sending 1 to $n$ to calculate

$$
\begin{aligned}
\sigma_{X N}(x \otimes n) & =\sigma_{X N}(X \otimes \hat{n})(x \otimes 1) \\
& =(\hat{n} \otimes X) \sigma_{X H}(x \otimes 1) \\
& =(\hat{n} \otimes X)\left(x_{(-1)} \otimes x_{(0)}\right) \\
& =x_{(-1)} n \otimes x_{(0)} .
\end{aligned}
$$

In particular,

$$
x_{(-1)} \otimes x_{(0)} r=\sigma(x \otimes 1) r=\sigma(x \otimes \bar{r} \cdot 1)=x_{(-1)} \bar{r} \otimes x_{(0)}
$$

so that $\delta_{0}$ takes values in $H \times_{R} X$. To prove that $\delta_{0}$ defines an $H$ comodule structure, we use the fact that comultiplication of $H$ is an 
$H$-module map $\Delta_{0}: H \rightarrow H \diamond H$ with respect to which $\sigma$ is natural. This yields the diagram

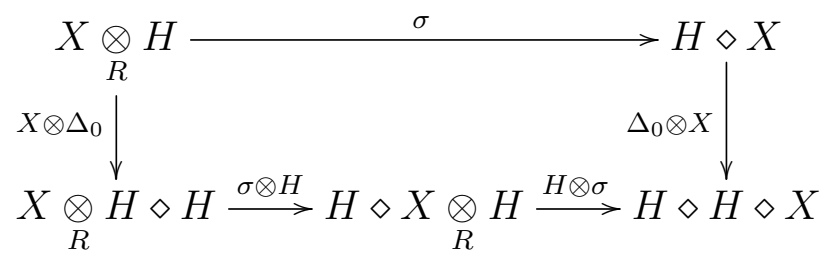

and chasing the element $x \otimes 1$ around this diagram yields

$$
\begin{aligned}
\Delta_{0}\left(x_{(-1)}\right) \otimes x_{(0)} & =\left(\Delta_{0} \otimes X\right) \sigma(x \otimes 1) \\
& =(H \otimes \sigma)(\sigma \otimes H)\left(X \otimes \Delta_{0}\right)(x \otimes 1) \\
& =x_{(-1)} \otimes x_{(0)(-1)} \otimes x_{(0)(0)} .
\end{aligned}
$$

We skip the details remaining to show that $X$ becomes an $H$-comodule and we have thus constructed the inverse functor to the claimed equivalence in $(2)$.

\section{Self-DUALity}

We will continue to consider the $\times$-Hopf algebra associated in section 4 to a sufficiently left rigid abelian monoidal category. We continue in the notations used in that section, but now we assume in addition that the category $\mathcal{C}$ is also sufficiently right rigid. We begin by proving once again the implication $(1) \Rightarrow 3$ of theorem 4.2 . Of course this is logically not necessary, but seems worthwhile since the proof avoids an awkward technical step.

So, we consider the monoidal functor $L: \mathcal{C} \rightarrow{ }_{A} \mathfrak{M}_{A}$, and use the fact that $L(X) \cong R(A) \otimes_{A} X$. Since $A$ is right rigid, its image under the rev-monoidal functor $R$ is left rigid in $\mathfrak{M}_{A}$, and thus finitely generated projective as right $A$-module. Exactly as in section 4 for $L(A)$ we can prove that $R(A)$ admits an epimorphism $R(A)^{n} \rightarrow A$ of $A$-bimodules, and thus $R(A)$ is a right $A$-progenerator. By the Morita theorems, $L$ factors over a category equivalence

$$
\begin{aligned}
\mathcal{C}={ }_{A} \mathfrak{M} & \longrightarrow{ }_{H} \mathfrak{M} \\
X & \longmapsto R(A){\underset{A}{\otimes}}_{X} X L(X)
\end{aligned}
$$

with, this time, $H:=\operatorname{End}_{-A}(R(A))$. Thus ${ }_{H} \mathfrak{M}$ is a monoidal category, and admits an underlying functor ${ }_{H} \mathfrak{M} \rightarrow{ }_{A}{ }^{\mathfrak{M}}$ that commutes with the underlying functors to the category of $k$-modules. By $\mid$ Sch98, Thm. 5.1, Rem. 5.3] we conclude that $H$ admits a $\times_{A}$-bialgebra structure for which the equivalence $A_{A} \mathfrak{M} \rightarrow{ }_{H} \mathfrak{M}$ is a monoidal equivalence. 
So far we have used two-sided sufficient rigidity to slightly simplify the proof in section 4, avoiding a last awkward passage to opposite rings. Now we will exploit it further to obtain a sort of self-duality.

Proposition 6.1. Let $\mathcal{C}$ be a sufficiently rigid cocomplete $k$-linear abelian monoidal category with right exact tensor products and a progenerator.

Then there is a $\times_{B}$-Hopf algebra $H$, finitely generated projective as left $\bar{B}$-module as well as left $R$-module, such that

$\mathcal{C} \cong{ }_{H} \mathfrak{M} \stackrel{\text { rev }}{\cong}{ }^{H} \mathfrak{M} \cong{ }_{H} \vee \mathfrak{M}, \quad H^{\text {cop }} \mathfrak{M} \cong{ }_{H} \vee \mathfrak{M}, \quad$ and $\quad{ }_{H} \mathfrak{M} \cong{ }_{\left(H^{\text {cop }}\right) \vee} \mathfrak{M}$ as monoidal categories.

Proof. By and large, the proof exploits the fact that $\mathcal{C}$ is a $\mathcal{C}$-bimodule category, and $\mathcal{C}$ is equivalent to the category of $k$-linear right exact right $\mathcal{C}$-endofunctors, as well as rev-equivalent to the category of $k$ linear right exact left $\mathcal{C}$-endofunctors of $\mathcal{C}$.

More concretely, the isomorphisms

$$
\begin{aligned}
& L(X) \underset{A}{\otimes} R(Y) \otimes_{A} Z \cong L(X) \underset{A}{\otimes}(Z \diamond Y) \cong X \diamond(Z \diamond Y) \cong \\
& \cong(X \diamond Z) \diamond Y \cong R(Y) \underset{A}{\otimes}(X \diamond Z) \cong R(Y) \underset{A}{\otimes} L(X) \underset{A}{\otimes_{A} Z}
\end{aligned}
$$

for $X, Y, Z \in \mathcal{C}$ induce isomorphisms

$$
\tau_{X Y}: R(X) \otimes_{A} L(Y) \rightarrow L(Y) \underset{A}{\otimes} R(X)
$$

that define monoidal functors $\hat{R}: \mathcal{C}^{\text {rev }} \rightarrow \mathcal{Z}(L)$ and $\hat{L}: \mathcal{C} \rightarrow \mathcal{Z}(R)$.

These two functors are category equivalences: For example, let $(M, \sigma) \in$ $\mathcal{Z}(L)$. Then $\sigma$ makes the endofunctor $M \otimes-$ of $\mathcal{C}$ commute with the left action of $\mathcal{C}$ on itself:

$$
\begin{aligned}
M{\underset{A}{\otimes}}_{(}(Y \diamond Z) & =M{\underset{A}{\otimes}}_{A} L(Y) \underset{A}{\otimes} Z \\
& \stackrel{\sigma}{\rightarrow} L(Y) \underset{A}{\otimes} M \otimes_{A} Z \\
& =Y \diamond\left(M \otimes_{A}^{\otimes} Z\right) .
\end{aligned}
$$

Therefore, $M \otimes_{A} Z \cong Z \diamond X$ for $X:=M \underset{A}{\otimes} I$, and we find $M \cong R(X)$, in such a way that $\sigma$ identifies with $\tau$.

Since $\mathcal{C}$ is ind-rigidly generated, the centralizers of $L$ and $R$ coincide with the left or right weak centralizers by lemma 3.8 .

Thus, the functor $R: \mathcal{C}^{\text {rev }} \rightarrow{ }_{A} \mathfrak{M}_{A}$ is the left weak centralizer of $L: \mathcal{C} \rightarrow{ }_{A} \mathfrak{M}$, and by proposition 5.1 we conclude that $R$ is a monoidal equivalence of $\mathcal{C}^{\text {rev }}$ with ${ }^{H} \mathfrak{M}$. 
By [Sch00, Cor. 5.15], the monoidal category of left $H$-comodules is equivalent to the category of left modules over the left dual $\times_{A}$-Hopf algebra.

Passing to coopposite $\times$-bialgebras corresponds to reversing tensor products in the module category.

For the last equivalence, we can apply the same reasoning replacing $H$ with $H^{\text {cop }}$.

Remark 6.2. In the situation of the preceding proposition, one is tempted to say that $H$ (or perhaps $H^{\text {cop }}$ ) is selfdual, because for ordinary Hopf algebras $H^{\vee}$ is just the opposite of the dual Hopf algebra, or the dual of the coopposite. However, $H^{\text {cop }}$ is a $\times_{A^{\text {op }}}$-bialgebra, so the statement that $H^{\text {cop }}$ is isomorphic to $H^{\vee}$ has no immediate meaning. We shall encounter situations below, however, where we can make sense of it.

Definition 6.3. Let $H$ be a $\times_{A}$-bialgebra.

If $\theta: A \rightarrow R$ is an isomorphism of algebras, then clearly $H$ can be

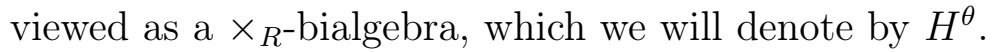

If $K$ is a $\times_{R^{-}}$-bialgebra, and $K \cong H^{\theta}$, as $\times_{R^{-}}$-bialgebras, then we will say that $K$ and $H$ are isomorphic.

If $H$ is finitely generated projective as left $\bar{A}$-module, and $H^{\text {cop }} \cong$ $H^{\vee}$, we shall say that $H$ is self-dual.

More generally, assume that $A$ and $R$ are Morita equivalent algebras, and fix an equivalence $\Theta:{ }_{A} \mathfrak{M} \rightarrow{ }_{R} \mathfrak{M}$ (given an isomorphism $\theta: A \rightarrow$ $R$ one could take $\Theta={ }_{\theta^{-1}} \mathfrak{M}$.) Then there is a monoidal category equivalence ${ }_{A} \mathfrak{M}_{A} \rightarrow{ }_{R} \mathfrak{M}_{R}$, which we denote by $\Theta$ again. It determines a $\times_{R^{-}}$bialgebra $H^{\Theta}$, obtained from $H$ by "Morita base change" [Sch03b|, such that we have an equivalence of monoidal categories ${ }_{H^{\Theta}} \mathfrak{M} \cong{ }_{H} \mathfrak{M}$ that commutes with the underlying functors to the respective categories of bimodules over the base as in the following diagram:

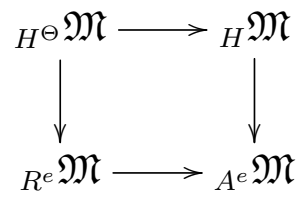

We shall say that $H$ is self-dual up to Morita base change if $H^{\text {cop }}$ is obtained from $H^{\vee}$ by Morita base change.

Remark 6.4. Note that if $H$ is a $\times_{A}$-bialgebra, then the algebra maps $A \rightarrow H$ and $\bar{A} \rightarrow H$ that make up the structure map $A \otimes \bar{A} \rightarrow H$ are injective.

Thus, an isomorphism $H \rightarrow K$ between a $\times_{A^{-}}$-bialgebra $H$ and a $\times_{R^{-}}$ bialgebra $K$ is the same thing as an isomorphism $f: H \rightarrow K$ of algebras such that $f(A)=R, f(\bar{A})=\bar{R}, f(\bar{a})=\overline{f(a)}, f\left(h_{(1)}\right) \otimes f\left(h_{(2)}\right)=$ 
$f(h)_{(1)} \otimes f(h)_{(2)}$, and $\epsilon(f(h))(f(a))=f(\epsilon(h)(a))$ for all $a \in A$ and $h \in H$.

Theorem 6.5. Let $A$ be a k-algebra, finitely generated projective over $k$, such that $\mathcal{C}={ }_{A} \mathfrak{M}$ is a $k$-linear abelian monoidal category. Assume that $\mathcal{C}$ is sufficiently rigid. Let $\mathcal{C}_{0} \subset \mathcal{C}$ be the subcategory of finitely generated projective objects. Let $J$ be a $k$-module, and consider the functor $F_{0}: \mathcal{C}_{0} \rightarrow \mathfrak{M}_{A}$ defined by $F_{0}(X)=\operatorname{Hom}_{k}\left({ }^{*} X, J\right)$.

If there is an isomorphism $\theta: A \rightarrow A^{\text {op }}$ and a natural isomorphism $F_{0}(X) \cong{ }_{\theta} X$, then $\mathcal{C}$ is monoidally equivalent to ${ }_{H} \mathfrak{M}$, where $H$ is a self-dual $\times_{B}$-Hopf algebra.

If $F_{0}$ extends to a $k$-linear category equivalence $F: \mathcal{C} \rightarrow \mathfrak{M}_{A}$, then $\mathcal{C}$ is equivalent to the monoidal category of modules over a $\times_{B}$-Hopf algebra $H$ which is self-dual up to Morita base change.

Proof. We start with the weaker assumption that $F_{0}$ extends to $F:{ }_{A} \mathfrak{M} \rightarrow$ $\mathfrak{M}_{A}$.

Write $D(X)=\operatorname{Hom}_{k}(X, J)$ so that $F_{0}(X)=D\left({ }^{*} X\right)$. If $X$ is a finitely generated projective, and hence left rigid object of $\mathcal{C}$, we have

$$
\begin{aligned}
D(X \diamond Y) & =\operatorname{Hom}_{k}(L(X) \underset{A}{\otimes} Y, J) \\
& =\operatorname{Hom}_{-A}\left(L(X), \operatorname{Hom}_{k}(Y, J)\right) \\
& =\operatorname{Hom}_{-A}(L(X), D(Y)) \\
& =D(Y) \otimes_{A} L\left(X^{*}\right) .
\end{aligned}
$$

and hence

$$
\begin{aligned}
F_{0}(X \diamond Y) & =D\left({ }^{*}(X \diamond Y)\right) \\
& =D\left({ }^{*} Y \diamond{ }^{*} X\right) \\
& =D\left({ }^{*} X\right){\underset{A}{\otimes}} L(Y) \\
& =F_{0}(X) \otimes_{A} L(Y) .
\end{aligned}
$$

Since every object of $\mathcal{C}$ is a colimit of finitely generated projective ones, we can extend this isomorphism to an isomorphism $F(X \diamond Y) \cong$ $F(X) \otimes L(Y)$ defined for arbitrary objects $X, Y$.

Denoting again $F$ the category equivalence ${ }_{A^{\text {op }}} \mathfrak{M}_{A^{\text {op }}} \rightarrow{ }_{A} \mathfrak{M}_{A}$ induced by $F$, we have thus shown that $F \circ L=R^{\text {op }}$, where $R^{\text {op }}(X)$ denotes $R(X)$, regarded as an $A^{\text {op }}$-bimodule. Now as $L: \mathcal{C} \rightarrow{ }_{A} \mathfrak{M}$ factors over the equivalence $\mathcal{C} \rightarrow{ }_{H} \mathfrak{M}$, the functor $F \circ L$ factors over an equivalence $\mathcal{C} \rightarrow{ }_{H^{F}} \mathfrak{M}$, where $H$ is obtained from $H$ by Morita base change. On the other hand we know from proposition 6.1 that $R: \mathcal{C}^{\text {rev }} \rightarrow{ }_{A} \mathfrak{M}^{\mathfrak{M} \text { factors }}$ 
over an equivalence $\mathcal{C}^{\text {rev }} \rightarrow H^{\vee} \mathfrak{M}$, which is the same as saying that $R^{\text {op }}$

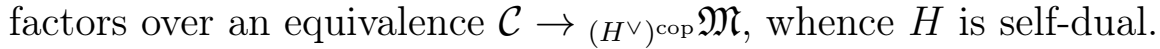

If the category equivalence $F$ is induced by an anti-automorphism of $A$, then we can infer that $H$ is self-dual rather than self-dual up to Morita base change.

Remark 6.6. If we require analogous hypotheses for the functor $F_{0}^{\prime}: \mathcal{C}_{0} \rightarrow$ $\mathfrak{M}_{A}$ defined by $F_{0}^{\prime}(X)=\operatorname{Hom}_{k}\left(X^{*}, J\right)$, we arrive at the conclusion that $H$ can be chosen so that $H^{\text {cop }}$ is self-dual (up to Morita base change).

Corollary 6.7. Let $A$ be a $k$-quasi-Hopf algebra finitely generated projective as $k$-module, whose antipode is bijective (the last condition is known to be void if $A$ is an ordinary Hopf algebra). Then $A_{A} \mathfrak{M}$ is monoidally equivalent to the category of modules over a self-dual $\times$ Hopf algebra.

Proof. The category $A \mathfrak{M}$ then satisfies the conditions of theorem 6.5. with $J=k$ and the map $\theta$ given by the antipode.

Remark 6.8. In the case just treated, the self-dual $\times$-Hopf algebra can be described more concretely. For $X \in A \mathfrak{M}$, the endofunctor $X \diamond-$ of ${ }_{A} \mathfrak{M}$ assigns $Y \in A_{A} \mathfrak{M}$ to the tensor product $X \otimes Y$ over $k$, with the diagonal module structure. But $\bullet \otimes \otimes \bullet Y \cong\left(\bullet X \otimes A_{\bullet}\right) \otimes_{A} Y$. Thus $L(X)=X \otimes A$, with the diagonal left $A$-module structure and the right $A$-module structure of the right tensor factor. Now by a result of Hausser and Nill [HN99] the functor $L$ factors over a category equivalence ${ }_{A} \mathfrak{M} \rightarrow{ }_{A} \mathfrak{M}_{A}^{A}$ with the category of Hopf modules, by endowing $L(X)=X \otimes A$ with the right comodule structure $\delta(x \otimes a)=$ $\phi^{(-1)} x \otimes \phi^{(-2)} a_{(1)} \otimes \phi^{(-3)} a_{(2)}$, where $\phi=\phi^{(-1)} \otimes \phi^{(-2)} \otimes \phi^{(-3)} \in A \otimes A \otimes A$ is the inverse of the coassociator of the quasi-Hopf algebra $A$. It was already observed by Hausser and Nill that this allows us to view $A \mathfrak{M}$ as the category of modules over a $\times_{A}$-bialgebra $H$ with coassociative comultiplication (while $A$ has non-coassociative comultiplication). The algebra $H$ whose modules are precisely the Hopf modules in $\mathfrak{M}_{A}^{A}$ is modelled on the $k$-module $A \otimes A \otimes A^{*}$; see [Sch03a. Constructions of associative algebras whose modules correspond to Hopf modules (with the same underlying $k$-module) go back to [CR98.

Corollary 6.9. Assume that $k$ is a field, $\underline{\mathcal{C}}$ is a rigid $k$-linear monoidal category, and equivalent to the category of finitely generated A-modules over a finite-dimensional $k$-algebra $A$. Then $\underline{\mathcal{C}}$ is monoidally equivalent to the category of finitely generated modules over a self-dual $\times$-Hopf algebra. This applies in particular if $\underline{\mathcal{C}}$ is a finite tensor category in the sense of [EO04], or the category of finitely generated modules over a weak Hopf algebra. 
Proof. We consider $\mathcal{C}={ }_{A} \mathfrak{M}$. Then $F_{0}$ as in theorem 6.5, with $J=k$, is defined on all of ${ }_{A} \mathfrak{m}=\underline{\mathcal{C}}$, giving an equivalence to the category of finitely generated right $A$-modules. Thus, the required extension $F$ always exists. Moreover, we can replace $A$ by a Morita equivalent basic algebra, and in this case the required isomorphism $\theta$ always exists.

The following corollary was proved by Pfeiffer $[\mathrm{Pfe} 09]$ under the additional hypothesis that the category in question is spherical.

Corollary 6.10. A fusion category is monoidally equivalent to the category of modules over a self-dual weak Hopf algebra.

Proof. The proof of theorem 6.5 provides us with a self-dual $\times_{B}$-Hopf algebra whose base $R$ is split semisimple. This is equivalent to a weak Hopf algebra, and self-duality as a $\times_{B}$-Hopf algebra clearly implies selfduality as a weak Hopf algebra (see $|\mathrm{Sch} 03 \mathrm{c}|$ for the translation between the formalisms of $\times$-Hopf algebras and weak Hopf algebras).

In the applications of theorem 6.5 so far, the $k$-module $J$ was always simply $k$ itself. In the following last version, we finally use a different choice. Admittedly, its hypotheses are not motivated by any specific applications beyond those already mentioned.

Corollary 6.11. Let $A$ be an artin k-algebra in the sense of [ARS95], that is, $k$ is an artinian ring, and $A$ is a $k$-algebra which is a finitely generated $k$-module. Assume that ${ }_{A} \mathfrak{m}$ is a $k$-linear abelian monoidal category in which projective as well as injective objects are rigid, in such a way that duals of projective (resp. injective) objects are injective (resp. projective). For example, this is the case if ${ }_{A} \mathfrak{m}$ is rigid.

Then ${ }_{A} \mathfrak{m}$ is monoidally equivalent to the category of modules over a self-dual $\times$-Hopf algebra.

Proof. Let $J$ be the injective envelope of $k$; then $D$ defines a duality between the module categories ${ }_{A} \mathfrak{m}$ and $\mathfrak{m}_{A}$, see ARS95, sec. II.3]. As taking duals in the category ${ }_{A} \mathfrak{m}$ provides a duality between projective and injective objects by assumption, we see that $F_{0}$ is an equivalence between finitely generated projective left $A$-modules and finitely generated projective right $A$-modules, which extends to an equivalence between all modules. Moreover we can replace $A$ by a Morita equivalent basic algebra, so that the isomorphism $\theta$ required in theorem 6.5 exists.

Guilty Plea 6.12. (1) If $H$ is obtained by Morita base change from a Hopf algebroid which is in turn self-dual, then $H$ is self-dual up to Morita base change. We do not know whether the converse is true. 
(2) The proof of self-duality up to Morita base change in theorem 6.5 relies on the hypothesis of a Morita equivalence between an algebra and its opposite, while self-duality requires an isomorphism between the algebra and its opposite.

Whether the distinction is really essential is related to an open purely ring-theoretic question |Fir13 : If a ring is Morita equivalent to its opposite, is it then Morita equivalent to a ring admitting an anti-automorphism? Since the answer is "yes" in a large class of examples, and no counterexample is known, the more general case treated in theorem 6.5 may in fact turn out not to be more general.

(3) We do not know of any examples of $\times_{B}$-bialgebras $H$ such that $H$ is self-dual, but not $H^{\mathrm{cop}}$. The same ignorance pertains to self-duality up to Morita base change.

\section{REFERENCES}

[ARS95] Maurice Auslander, Idun Reiten, and Sverre O. Smalø. Representation theory of Artin algebras. Volume 36 of Cambridge Studies in Advanced Mathematics. Cambridge University Press, Cambridge, 1995, pages xiv +423 .

[BLV11] Alain Bruguières, Steve Lack, and Alexis Virelizier. Hopf monads on monoidal categories. Adv. math., 227(2):745800, 2011.

[Böh09] Gabriella Böhm. Hopf algebroids. In, Handbook of algebra. Vol. 6. Volume 6, in Handb. Algebr. Pages 173-235. Elsevier/NorthHolland, Amsterdam, 2009.

[CR98] Claude Cibils and Marc Rosso. Hopf bimodules are modules. J. pure appl. algebra, 128(3):225-231, 1998.

[ENO05] Pavel Etingof, Dmitri Nikshych, and Viktor Ostrik. On fusion categories. Ann. of math. (2), 162(2):581-642, 2005.

[EO04] Pavel Etingof and Viktor Ostrik. Finite tensor categories. Mosc. math. j., 4(3):627-654, 782-783, 2004.

[Fir13] U. A. First. Rings That Are Morita Equivalent to Their Opposites. Arxiv e-prints, May 2013. arXiv: 1305.5139 [math.RA].

[Hái02] Phùng Hô Hái. An embedding theorem for abelian monoidal categories. Compositio math., 132(1):27-48, 2002.

[Hái08] Phùng Hô Hái. Tannaka-Krein duality for Hopf algebroids. Israel j. math., 167:193-225, 2008. 
[Hay99] Takahiro Hayashi. A canonical Tannaka duality for finite seimisimple tensor categories. Arxiv mathematics e-prints, April 1999. eprint: math/9904073.

[HN99] F. Hausser and F. Nill. Integral Theory for Quasi-Hopf Algebras. Arxiv mathematics e-prints, April 1999. eprint: math/9904164.

[Kas95] Christian Kassel. Quantum groups. Volume 155 of Graduate Texts in Mathematics. Springer-Verlag, New York, 1995, pages xii +531 .

[Maj91] Shahn Majid. Representations, duals and quantum doubles of monoidal categories. In Proceedings of the Winter School on Geometry and Physics (Srni, 1990). (26), 1991, pages 197-206.

[Mit65] Barry Mitchell. Theory of categories. Of Pure and Applied Mathematics, Vol. XVII. Academic Press, New York, 1965, pages $\mathrm{xi}+273$.

[Müg03a] Michael Müger. From subfactors to categories and topology. I. Frobenius algebras in and Morita equivalence of tensor categories. J. pure appl. algebra, 180(1-2):81-157, 2003.

[Müg03b] Michael Müger. From subfactors to categories and topology. II. The quantum double of tensor categories and subfactors. J. pure appl. algebra, 180(1-2):159-219, 2003.

[Müg10] Michael Müger. Tensor categories: a selective guided tour. Rev. un. mat. argentina, 51(1):95-163, 2010.

[Ost03] Victor Ostrik. Module categories, weak Hopf algebras and modular invariants. Transform. groups, 8(2):177-206, 2003.

[Par77] Bodo Pareigis. Non-additive ring and module theory II. $\mathcal{C}$ categories, $\mathcal{C}$-functors and $\mathcal{C}$-morphisms. Publ. math. debrecen, 24:351-361, 1977.

[Pfe09] Hendryk Pfeiffer. Finitely semisimple spherical categories and modular categories are self-dual. Adv. math., 221(5):16081652, 2009.

[Sch00] Peter Schauenburg. Duals and doubles of quantum groupoids $\left(\times_{R}\right.$-Hopf algebras). In, New trends in hopf algebra theory (la falda, 1999). Volume 267, in Contemp. Math. Pages 273299. Amer. Math. Soc., Providence, RI, 2000.

[Sch03a] Peter Schauenburg. Actions of monoidal categories and generalized Hopf smash products. J. algebra, 270(2):521-563, 2003. 
[Sch03b] Peter Schauenburg. Morita base change in quantum groupoids. In, Locally compact quantum groups and groupoids (strasbourg, 2002). Volume 2, in IRMA Lect. Math. Theor. Phys. Pages 79-103. de Gruyter, Berlin, 2003.

[Sch03c] Peter Schauenburg. Weak Hopf algebras and quantum groupoids. In, Noncommutative geometry and quantum groups (warsaw, 2001). Volume 61, in Banach Center Publ. Pages 171188. Polish Acad. Sci., Warsaw, 2003.

[Sch98] Peter Schauenburg. Bialgebras over noncommutative rings and a structure theorem for Hopf bimodules. Appl. categ. structures, 6(2):193-222, 1998.

[Szl05] Kornel Szlachanyi. Adjointable monoidal functors and quantum groupoids. In, Hopf algebras in noncommutative geometry and physics. Volume 239, in Lecture Notes in Pure and Appl. Math. Pages 291-307. Dekker, New York, 2005.

[Tak77] Mitsuhiro Takeuchi. Groups of algebras over $A \otimes \bar{A}$. J. math. soc. japan, 29(3):459-492, 1977.

Institut de Mathématiques de Bourgogne, Université de Bourgogne, Faculté des Sciences Mirande, 9 avenue Alain Savary, BP 4787021078 Dijon Cedex, France

E-mail address: peter.schauenburg@u-bourgogne.fr 